\title{
Estado nutricional, factores sociodemográficos y de salud en estudiantes de nuevo ingreso a la UAZ
}

\section{Nutritional status and social-demographical and health factors among newly enrolled students at UAZ}

\section{Estado nutricional, fatores sociodemográficos e de saúde em estudantes de novo ingresso à UAZ}

\author{
D. Manzanero-Rodríguez ${ }^{\mathrm{a}}$, A.M. Rodríguez Rodríguez ${ }^{\mathrm{a}}$, L. García-Esquivel ${ }^{\mathrm{b}}$, J.M. Cortez-Solís ${ }^{\mathrm{b}}$ \\ ${ }^{a}$ Licenciatura en Nutrición, Universidad Autónoma de Zacatecas, Zacatecas, México

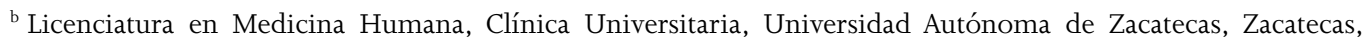 \\ México
}

Recibido: 5 enero 2018

Aceptado: 2 septiembre 2018

\section{Resumen}

Introducción: El sobrepeso y obesidad son factores de riesgo para desarrollar complicaciones a corto y largo plazo. La población de nuevo ingreso a la universidad se considera un colectivo, especialmente, vulnerable desde el punto de vista nutricional.

Objetivo: Describir el estado nutricional, factores sociodemográficos y de salud en estudiantes de nuevo ingreso a la UAZ.

Metodología: Estudio de tipo observacional, transversal y descriptivo, que se realizó a 3,972 estudiantes universitarios de nuevo ingreso. Se logró el consentimiento verbal de los alumnos. Se capacitaron y estandarizaron a los pasantes de enfermería, medicina y nutrición, para llevar a cabo las mediciones. Se obtuvo información del estado nutricional, factores sociodemográficos y salud, así como mediciones antropométricas.

Resultados: Los hombres tienen mayor prevalencia de sobrepeso $(24.1 \%)$ y obesidad (9.2\%) que las mujeres $(p<0.001)$. Los hombres realizan mayor actividad física $(73.6 \%$ vs $51.1 \%)$, consumen más alcohol $(58.3 \%$ vs $34.3 \%)$ y tabaco $(20.8 \%$ vs $9.5 \%)$ ( $p<0.000)$. Se encontró una asociación positiva en los momios de sobrepeso en relación con los hombres ( $\mathrm{RM}=1.22$, IC 95\% 1.02-1.45), edad de 19 años ( $\mathrm{RM}=1.36$, IC 95\% 1.02-1.45), en las áreas de ciencias de la salud (RM=1.88, IC 95\% 1.05-3.35), ciencias sociales ( $\mathrm{RM}=1.93$, IC 95\% 1.06-3.48), humanidades y educativas ( $\mathrm{RM}=1.90$, IC 95\% 1.01-3.53), ingenierías y tecnologías ( $\mathrm{RM}=1.83$, IC 95\% 1.01-3.30).

\footnotetext{
*Autor de correspondencia. Correo electrónico: daniel81_manzanero@hotmail.com http: //dx.doi.org/10.22201/eneo.23958421e.2018.4.545 
Discusión y conclusión: Se puede contribuir a reducir las prevalencias de sobrepeso y obesidad de los estudiantes, a través de intervenciones dirigidas a modificar las conductas de riesgo durante la estancia universitaria, mejorar la alimentación y promover estilos de vida saludables.

Palabras clave: Sobrepeso; obesidad; estudiantes universitarios; estilos de vida saludable; hábitos; estado nutricional; México.

\section{Abstract}

Introduction: Obesity and overweight are risk factors to developing short and long-term health-related complications; and newly enrolled university students are considered a vulnerable group in terms of their nutritional progression status.

Objective: To describe the nutritional status, and social-demographical and health factors among newly enrolled students at UAZ.

Methodology: This is an observational, transversal, and descriptive study on a sample of 3,972 newly enrolled university students who verbally consented on their participation. Nursing, medicine, and nutrition intern students were trained to carry out diverse assessments related to the nutritional status, social-demographical and health factors, and anthropometric data on the sample.

Results: Newly enrolled male students showed a higher prevalence of overweight (24.1\%) and obesity $(9.2 \%)$ in comparison to their female counterparts $(\mathrm{p}<.001)$. Male students showed having more physical activity (73.6\% vs $51.1 \%$ ) but also consuming more alcohol (58.3\% vs $34.3 \%)$ and tobacco $(20.8 \%$ vs $9.5 \%)(\mathrm{p}<.000)$. Positive associations were found between the overweight numbers and, being male $(\mathrm{RM}=1.22$, CI 95\% 1.02-1.45), being 19 years old $(\mathrm{RM}=1.36$, CI 95\% 1.02-1.45), being in the areas of health sciences ( $\mathrm{RM}=1.88$, CI 95\% 1.05-3.35), being in the areas of social sciences ( $\mathrm{RM}=1.93$, CI 95\% 1.06-3.48), being in the areas of humanities and education ( $\mathrm{RM}=1.90$, CI 95\% 1.01-3.53), and being in the areas of engineering and technology ( $\mathrm{RM}=1.83$, CI 95\% 1.01-3.30).

Discussion and conclusion: It is necessary to address to obesity and overweight problems among university students through interventions aimed at modifying risky behaviors during their university stay, improving the quality of nutrition, and promoting healthy life-styles.

Keywords: Overweight; obesity; university students; healthy life-styles; habits; nutritional status; Mexico.

\section{Resumo}

Introdução: O sobrepeso e a obesidade são fatores de risco para desenvolver complicações a curto e longo prazo. A população de novo ingresso à universidade considera-se um coletivo, especialmente, vulnerável desde o ponto de vista nutricional.

Objetivo: Descrever o estado nutricional, fatores sociodemográficos e de saúde em estudantes de novo ingresso à UAZ.

Metodologia: Estudo de tipo observacional, transversal e descritivo, que se realizou a 3,972 estudantes universitários de novo ingresso. Conseguiu-se o consentimento verbal dos alunos. Capacitaram-se e padronizaram os formados de enfermagem, medicina e nutrição, para levar a cabo as medições. Obteve-se informação do estado nutricional, fatores sociodemográficos e saúde, assim como medições antropométricas.

Resultados: Os homens de novo ingresso têm maior prevalência de sobrepeso (24.1\%) e obesidade $(9.2 \%)$ quanto as mulheres $(\mathrm{p}<0.001)$. Homens realizam mais atividade física que mulheres $(73.6 \%$ vs. $51.1 \%)$, consomem mais álcool ( $58.3 \%$ vs. $34.3 \%)$ e tabaco (20.8\% vs. 9.5\%) (p <0.000). Encontrou-se uma associação positiva nas chances de sobrepeso em relação com aos homens (RM $=1.22$, IC $95 \% 1.02$ $1.45)$, ter 19 anos de idade $(\mathrm{RM}=1.36$, IC $95 \% 1.02-1.45)$ nas áreas de ciências da saúde $(\mathrm{RM}=1.88$, IC $95 \% 1.05-3.35)$, ciências sociais $(\mathrm{RM}=1.93$, IC 95\% 1.06-3.48), humanidades e educativas ( $\mathrm{RM}=$ 1.90, IC 95\% 1.01-3.53), engenharias e tecnologias ( $\mathrm{RM}=1.83$, IC 95\% 1.01-3.30). 
Discussão e conclusão: Pode-se contribuir a reduzir as prevalências de sobrepeso e obesidade dos estudantes, a través de intervenções dirigidas a modificar as condutas de risco durante a permanência universitária, melhorar a alimentação e promover estilos de vida saudáveis.

Palavras chave: Sobrepeso; obesidade; estudantes universitarios; estilos de vida saudáveis; hábitos; estado nutricional; México

\section{Introducción}

La obesidad es un factor de riesgo para la aparición de enfermedades crónicas no transmisibles (ECNT), como son: la hipertensión, enfermedades cardiovasculares, padecimientos respiratorios crónicos, diabetes, cáncer de colon y de mama, así como las dislipidemias ${ }^{1}$. Del total de muertes a nivel mundial, casi dos tercios se debe a enfermedades crónicas no transmisibles ${ }^{2,3}$. El desencadenamiento de estas enfermedades se debe, principalmente, a una reducción gradual de la actividad física que tiene relación con los siguientes factores: 1) sociodemográficos (género femenino, mayor edad, mayores ingresos), 2) psicológicos (baja autoestima), 3) sociales (falta de apoyo social, sentido de responsabilidad), 4) comportamiento de riesgo para la salud (fumar, consumir alcohol, tener sobrepeso u obesidad, consumo insuficiente de frutas y verduras), 5) síntomas depresivos y 6) falta de conocimiento del vínculo ejercicio-enfermedad cardíaca y del ejercicio en los beneficios para la salud ${ }^{4}$, todo esto acompañado por un aumento en la ingesta de energía en la dieta 5 .

Se estima que en el mundo existe un billón de adultos con sobrepeso (Índice de Masa Corporal-IMCentre 25-29.9) ${ }^{6}$, de los cuales 310 millones pueden ser clasificados como obesos (IMC $\geq 30 \mathrm{~kg} / \mathrm{m}^{2}$ ). Se ha proyectado que para el año 2030 el número de personas obesas aumentaría a 360 millones de personas en todo el mundo. En países desarrollados como Estados Unidos, la prevalencia de obesidad en adultos ha incrementado entre 1999 y 2010, hasta alcanzar 35.5\% en hombres y 35.8\% en mujeres en 20092010. A su vez, en países europeos como España, la prevalencia de sobrepeso en adultos es $34.2 \%$, con una mayor proporción en hombres que en mujeres, (43.9\% y $25.7 \%$, respectivamente) ${ }^{7}$.

En México, de acuerdo a los resultados de la Encuesta Nacional de Salud y Nutrición ENSANUT 2016, en el grupo de adolescentes de 12 a 19 años de edad la prevalencia combinada de sobrepeso y obesidad fue de $36.3 \%$. En 2012, esta cifra era de 34.9\%. En adultos mayores de 20 años de edad la prevalencia combinada de sobrepeso y obesidad fue de $72.5 \%{ }^{8}$.

Durante las últimas décadas la población general de los países desarrollados y en vías de desarrollo ha experimentado un proceso de transición epidemiológica y nutricional ${ }^{9}$, con el resultado de cambios marcados en los patrones de consumo de alimentos y comportamientos de estilo de vida. Los hábitos alimentarios tradicionales han sido reemplazados por otros más occidentalizados, que se caracterizan por una baja ingesta de fibra dietética, verduras y frutas, y un alto consumo de alimentos ricos en grasas, azúcar y sal.

De esta manera, la población que recién ingresa a la universidad, (adolescencia tardía y edad adulta temprana), que comprende un período de 18 a 24 años de edad ${ }^{10}$, se considera un colectivo, especialmente, vulnerable desde el punto de vista nutricional ${ }^{11}$, ya que a menudo comienzan a vivir por primera vez lejos de casa de los padres, obtienen más libertad y hacen nuevos amigos ${ }^{12}$, por lo que comienzan a responsabilizarse de su alimentación y atraviesan un período crítico en la consolidación de hábitos y conductas alimentarias, importantes para la salud futura ${ }^{13,14}$.

Las comidas rápidas, que generalmente contienen una gran cantidad de calorías, grasa y sal, se convertirán en artículos esenciales en las comidas de los estudiantes.

Este aumento de energía es debido al incremento en el consumo de alimentos ricos en grasas y en carbohidratos refinados; bajos en fibra y reducidos en micronutrientes, los cuales son hábitos recientes de alimentación. Los estudiantes que ingresan a la universidad son uno de los sectores poblacionales con mayor nivel educativo de un país, no obstante, aparentemente no escapan al problema antes mencionado ${ }^{15}$. 
El estilo de vida se ha definido como el conjunto de pautas y hábitos comportamentales cotidianos de una persona y como aquellos patrones de conducta individuales que demuestran cierta consistencia en el tiempo, bajo condiciones más o menos constantes y que pueden constituirse en dimensiones de riesgo o de seguridad dependiendo de su naturaleza. Aunque, lógicamente sería difícil encontrar conductas humanas que no tuvieran algún tipo de influencia sobre la salud y el bienestar, existe en la actualidad una serie de repertorios de conducta que se encuentran entre los factores de riesgo más importantes, tales como, el desequilibrio en la dieta, el sedentarismo, el consumo de tabaco y de alcohol, no dormir el tiempo necesario ${ }^{16}$, no practicar ejercicio físico, no participar en programas de promoción de la salud, incumplir las instrucciones médicas y utilizar de forma inadecuada los servicios de salud, entre otros ${ }^{17}$.

Ante esta situación, se requiere un análisis más profundo con la información obtenida del examen médico de ingreso a la Universidad Autónoma de Zacatecas (UAZ), para plantear alternativas que den solución a los problemas de relevancia, que presentan los jóvenes de recién ingreso a la máxima casa de estudios del estado de Zacatecas.

Por lo tanto, el objetivo del presente estudio fue describir el estado nutricional, factores sociodemográficos y estilos de vida en estudiantes universitarios de nuevo ingreso a la Universidad Autónoma de Zacatecas.

\section{Metodología}

Se llevó a cabo un estudio de tipo transversal, observacional y descriptivo. De un total de 5,000 estudiantes de nuevo ingreso a las licenciaturas e ingenierías de la UAZ, se logró recopilar información completa de 3,972 (80\% del total de alumnos) estudiantes de nuevo ingreso a 33 licenciaturas e ingenierías, divididas en siete áreas académicas (Ciencias de la salud, Arte y cultura, Ciencias sociales, Ciencias Socio-Políticas Económicas y Administrativas, Humanidades y educación, Ingenierías y tecnologías, y Ciencias básicas). Una vez que fueron aceptados para ingresar a la Universidad, se les solicitó trasladarse a la clínica universitaria de la UAZ para realizarles el examen médico general. Se les pidió que asistieran sin accesorios, aretes, reloj, en ayuno de por lo menos 6 horas, con ropa ligera y, las mujeres, que no estuvieran en su periodo menstrual. El examen médico se realizó de junio a septiembre de 2015, por muestreo no probabilístico consecutivo ${ }^{18}$. Se solicitó el consentimiento verbal de los alumnos después de explicarles en qué consistía el examen médico y cuáles eran los objetivos. Los médicos que realizaron las encuestas capacitaron y estandarizaron a los pasantes de nutrición, enfermería y medicina, para que llevaran a cabo las mediciones clínicas y antropométricas. Se excluyeron las historias clínicas que presentaron datos incongruentes o incompletos. El estudio fue autorizado por el comité de ética de la clínica universitaria de la UAZ. Se obtuvo información del estado nutricional a través de IMC con el cual se clasificó a los participantes según las categorías propuestas por la OMS, para el diagnóstico de sobrepeso (IMC $\geq 25$ y $<30$ ) y obesidad (IMC $\geq 30)^{19}$; se recopiló información sobre factores sociodemográficos mediante género, edad, estado civil, procedencia, área académica, unidad de ingreso del estudiante y escolaridad del tutor; estilos de vida con base en la actividad física, consumo de alcohol y tabaco. El material utilizado fue una Tanita modelo BC-533, que contiene cuatro memorias para usuarios diferentes, pantalla LCD, capacidad de 150 $\mathrm{kg}$, incremento de peso en $100 \mathrm{f}$. Estadímetro marca SECA modelo 220: rango de medición de 60-200 cm.

Se analizó la información con el software estadístico STATA versión 14.0. Se consideró que un valor de $\mathrm{p}<0.05$ era estadísticamente significativo ${ }^{20}$.

\section{Resultados}

Se obtuvo un total de 3,972 historias clínicas con datos válidos de los cuales 2,232 fueron mujeres, que representan el $56.1 \%$ de la muestra ( $20.1 \pm 5.1$ años) y 1,740 fueron hombres, que representan el $43.8 \%$ de la muestra (19.8 años \pm 4.3 años). Se agruparon en 3 categorías de edad de $\leq 17$ años $(9.3 \%)$, 18 años (47.8\%) y $\geq 19$ años (42.8\%). Se logró obtener información de 33 de las 40 licenciaturas que ofrece la UAZ, divididas en sus siete áreas académicas, con mayor representatividad del área de ciencias 
de la salud (34.8\%), seguida del área de ingenierías y tecnologías (20.3\%), así como de las áreas de ciencias sociales $(15.8 \%)$ y ciencias socio-políticas económicas y administrativas (14.3\%). Los hombres predominan en el área de ingenierías y tecnologías (36.5\%), seguido del área de ciencias de la salud (25.5\%). Por su parte, las mujeres tienen mayor presencia en áreas de: ciencias de la salud (42.2\%), ciencias sociales $(19.0 \%)$ y de ciencias socio-políticas económicas y administrativas (16.7\%). Con respecto al estado civil, el 93.5\% de los estudiantes se encontraban solteros al momento de la entrevista (Hombres 95.7\%; Mujeres 91.9\%). De acuerdo a la procedencia de los estudiantes, 1,830 (46.0\%) provienen del área conurbada Zacatecas-Guadalupe, 1,979 (49.8\%) proceden de los 56 municipios restantes del estado de Zacatecas, y los demás alumnos son de otros estados o del extranjero (Tabla 1).

Tabla 1. Características generales de los alumnos de nuevo ingreso según género, Universidad Autónoma de Zacatecas 2015 ( $n=3972)$

\begin{tabular}{|c|c|c|c|c|c|}
\hline & \multicolumn{4}{|c|}{ Género } & \multirow[b]{3}{*}{$\mathbf{p}$} \\
\hline & \multicolumn{2}{|c|}{ Hombre } & \multicolumn{2}{|c|}{ Mujer } & \\
\hline & $\mathbf{F}$ & $\%$ & $\mathbf{F}$ & $\%$ & \\
\hline \multicolumn{6}{|l|}{ Edad } \\
\hline$\leq 17$ años & 147 & 8.4 & 226 & 10.1 & $0.028 *$ \\
\hline 18 años & 811 & 46.6 & 1,088 & 48.7 & \\
\hline$\geq 19$ años & 782 & 44.9 & 918 & 41.1 & \\
\hline \multicolumn{6}{|l|}{ Áreas académicas } \\
\hline Ciencias de la salud & 444 & 25.5 & 941 & 42.2 & $0.000 *$ \\
\hline Arte y cultura & 56 & 3.2 & 63 & 2.8 & \\
\hline Ciencias sociales & 206 & 11.8 & 423 & 19.0 & \\
\hline Cs. Sociopol, económ. y admvas. & 198 & 11.4 & 372 & 16.7 & \\
\hline Humanidades y educativas & 130 & 7.5 & 181 & 8.1 & \\
\hline Ingenierías y tecnologías & 635 & 36.5 & 174 & 7.8 & \\
\hline Ciencias básicas & 71 & 4.1 & 78 & 3.5 & \\
\hline \multicolumn{6}{|l|}{ Estado civil } \\
\hline Casado & 62 & 3.6 & 140 & 6.3 & $0.000 *$ \\
\hline Divorciado & 4 & 0.2 & 14 & 0.6 & \\
\hline Soltero & 1,665 & 95.7 & 2,051 & 91.9 & \\
\hline Unión libre & 7 & 0.4 & 23 & 1.0 & \\
\hline Viudo & 2 & 0.1 & 4 & 0.2 & \\
\hline \multicolumn{6}{|l|}{ Procedencia } \\
\hline Zacatecas-Guadalupe & 820 & 47.1 & 1,010 & 45.3 & 0.107 \\
\hline Municipios de Zacatecas & 847 & 48.7 & 1,132 & 50.7 & \\
\hline Otros estados & 67 & 3.9 & 72 & 3.2 & \\
\hline Extranjeros & 6 & 0.3 & 18 & 0.8 & \\
\hline \multicolumn{6}{|l|}{ Escolaridad del padre o tutor } \\
\hline Primaria & 291 & 17.0 & 406 & 18.6 & $0.002 *$ \\
\hline Secundaria & 408 & 23.9 & 603 & 27.6 & \\
\hline Preparatoria & 423 & 24.7 & 447 & 20.4 & \\
\hline Licenciatura & 589 & 34.4 & 732 & 33.5 & \\
\hline
\end{tabular}

P: chi cuadrada de Pearson; *Significancia estadística.

En lo que concierne a la práctica de actividad física y estilos de vida no saludables (Tabla 2) se encontró que los hombres realizan mayor actividad física que las mujeres (73.6\% vs $51.1 \%)$, consumen más alcohol (58.3\% vs $34.3 \%)$ y tabaco $(20.8 \%$ vs $9.5 \%)$, todas las diferencias entre variables tienen significancia estadística $(p<0.000)$. 
Tabla 2. Práctica de actividad física y estilos de vida no saludables de los alumnos de nuevo ingreso según género, Universidad Autónoma de Zacatecas 2015 ( $n=3972$ )

\begin{tabular}{|c|c|c|c|c|c|}
\hline & \multicolumn{4}{|c|}{ Género } & \multirow[b]{3}{*}{$\mathbf{P}$} \\
\hline & \multicolumn{2}{|c|}{ Hombre } & \multicolumn{2}{|c|}{ Mujer } & \\
\hline & $\mathbf{F}$ & $\%$ & $\mathbf{F}$ & $\%$ & \\
\hline \multicolumn{6}{|c|}{ Actividad física } \\
\hline $\mathrm{Si}$ & 1,280 & 73.6 & 1,141 & 51.1 & $0.000 *$ \\
\hline No & 460 & 26.4 & 1,091 & 48.9 & \\
\hline \multicolumn{6}{|c|}{ Consumo de alcohol } \\
\hline $\mathrm{Si}$ & 988 & 58.3 & 740 & 34.3 & $0.000^{*}$ \\
\hline No & 707 & 41.7 & 1,419 & 65.7 & \\
\hline \multicolumn{6}{|c|}{ Consumo de tabaco } \\
\hline $\mathrm{Si}$ & 346 & 20.8 & 203 & 9.5 & $0.000^{*}$ \\
\hline No & 1,321 & 79.2 & 1,936 & 90.5 & \\
\hline
\end{tabular}

P: chi cuadrada de Pearson; *Significancia estadística.

Alrededor de dos terceras partes de la población quedaron clasificadas con peso bajo o peso normal, y $30.6 \%$ en sobrepeso u obesidad; los hombres presentaron un porcentaje mayor de sobrepeso y obesidad $(24.1 \%$ y $9.2 \%)$ que las mujeres $(20.7 \%$ y $7.8 \%)$ del estudio $(p<0.001)$. Por otro lado, las prevalencias más elevadas de sobrepeso se observaron en la diferenciación según estado civil, y correspondió a quienes estaban divorciados $(61.1 \%)$ y casados $(40.1 \%)$. En cuanto a las variables de prácticas vinculadas con la salud, quienes no realizaban actividad física tienen mayor prevalencia de sobrepeso $(23.0 \%)$ y obesidad ( $8.5 \%)$ respecto a los que la realizan $(21.7 \%$ y $8.4 \%$ respectivamente). También es mayor el sobrepeso y obesidad en quienes consumían alcohol (24.3\% y 9.3\%) y quienes fumaban (26.5\% y $9.8 \%$ ), todas las variables anteriores presentaron significancia estadística $(p<0.001)$ (Tabla 3).

Tabla 3. Estado nutricional según variables sociodemográficas y estilos de vida de los alumnos de nuevo ingreso a las licenciaturas de la UAZ, 2015 (n=3972)

\begin{tabular}{|c|c|c|c|c|c|c|c|c|c|}
\hline & \multicolumn{8}{|c|}{ Clasificación del estado nutricional } & \multirow[b]{3}{*}{$\mathbf{p}$} \\
\hline & \multicolumn{2}{|c|}{ Bajo peso } & \multicolumn{2}{|c|}{ Normal } & \multicolumn{2}{|c|}{ Sobrepeso } & \multicolumn{2}{|c|}{ Obesidad } & \\
\hline & $\mathbf{F}$ & $\%$ & $\mathbf{F}$ & $\%$ & $\mathbf{F}$ & $\%$ & $\mathbf{F}$ & $\%$ & \\
\hline \multicolumn{10}{|l|}{ Género } \\
\hline Hombre & 132 & 7.5 & 1,025 & 58.9 & 420 & 24.1 & 161 & 9.2 & $0.001 *$ \\
\hline Mujer & 234 & 10.5 & 1,358 & 60.9 & 463 & 20.7 & 174 & 7.8 & \\
\hline \multicolumn{10}{|l|}{ Edad } \\
\hline$\leq 17$ años & 45 & 12.0 & 232 & 62.2 & 73 & 19.5 & 23 & 6.1 & $0.000 *$ \\
\hline 18 años & 207 & 10.9 & 1,200 & 63.3 & 362 & 19.1 & 126 & 6.6 & \\
\hline$\geq 19$ años & 114 & 6.7 & 951 & 55.9 & 448 & 26.3 & 186 & 10.9 & \\
\hline \multicolumn{10}{|l|}{ Procedencia } \\
\hline Zac.-Guadalupe & 155 & 8.4 & 1,061 & 58.1 & 425 & 23.2 & 184 & 10.1 & $0.000 *$ \\
\hline Estado Zac. & 195 & 9.8 & 1,229 & 62.1 & 415 & 20.9 & 140 & 7.0 & \\
\hline Otros estados & 11 & 7.9 & 86 & 61.8 & 32 & 23.0 & 10 & 7.1 & \\
\hline Extranjeros & 5 & 20.8 & 7 & 29.1 & 11 & 45.8 & 1 & 4.1 & \\
\hline \multicolumn{10}{|l|}{ Estado civil } \\
\hline Casado & 5 & 2.4 & 82 & 40.5 & 81 & 40.1 & 34 & 16.8 & $0.000 *$ \\
\hline Divorciado & 0 & 0.0 & 4 & 22.2 & 11 & 61.1 & 3 & 16.6 & \\
\hline Soltero & 356 & 9.5 & 2,278 & 61.3 & 785 & 21.1 & 292 & 7.8 & \\
\hline
\end{tabular}


Tabla 3. Continuación.

\begin{tabular}{|c|c|c|c|c|c|c|c|c|c|}
\hline & \multicolumn{8}{|c|}{ Clasificación del estado nutricional } & \multirow[b]{3}{*}{$\mathbf{p}$} \\
\hline & \multicolumn{2}{|c|}{ Bajo peso } & \multicolumn{2}{|c|}{ Normal } & \multicolumn{2}{|c|}{ Sobrepeso } & \multicolumn{2}{|c|}{ Obesidad } & \\
\hline & $\mathbf{F}$ & $\%$ & $\mathbf{F}$ & $\%$ & $\mathbf{F}$ & $\%$ & $\mathbf{F}$ & $\%$ & \\
\hline Unión libre & 4 & 13.3 & 17 & 56.6 & 5 & 16.6 & 4 & 13.3 & \\
\hline Viudo & 1 & 16.6 & 2 & 33.3 & 1 & 16.6 & 2 & 33.3 & \\
\hline \multicolumn{10}{|l|}{ Actividad física } \\
\hline $\mathrm{Si}$ & 183 & 7.5 & 1,505 & 62.2 & 526 & 21.7 & 203 & 8.4 & $0.000 *$ \\
\hline No & 183 & 11.8 & 878 & 56.6 & 357 & 23.0 & 132 & 8.5 & \\
\hline \multicolumn{10}{|c|}{ Consumo de alcohol } \\
\hline $\mathrm{Si}$ & 113 & 6.5 & 1,033 & 59.7 & 421 & 24.3 & 161 & 9.3 & $0.000 *$ \\
\hline No & 240 & 11.3 & 1,279 & 60.3 & 442 & 20.8 & 160 & 7.5 & \\
\hline \multicolumn{10}{|c|}{ Consumo de tabaco } \\
\hline $\mathrm{Si}$ & 33 & 6.0 & 316 & 57.5 & 146 & 26.5 & 54 & 9.8 & $0.001 *$ \\
\hline No & 321 & 9.8 & 1,970 & 60.5 & 696 & 21.4 & 265 & 8.1 & \\
\hline
\end{tabular}

P: chi cuadrada de Pearson; *Significancia estadística.

Finalmente, se construyó un modelo multivariado con la variable principal de estudio que fue el sobrepeso, se utilizó como covariables el sexo, edad, áreas académicas, procedencia, actividad física, consumo de tabaco y alcohol. Se encontró una asociación positiva en los momios de sobrepeso en relación con los hombres ( $\mathrm{RM}=1.22$, IC 95\% 1.02-1.45), en el grupo de edad de 19 años ( $R M=1.36$, IC 95\% 1.02-1.45), en las áreas académicas de ciencias de la salud ( $\mathrm{RM}=1.88$, IC 95\% 1.05-3.35), ciencias sociales ( $R M=1.93$, IC 95\% 1.06-3.48), humanidades y educativas ( $R M=1.90$, IC 95\% 1.01-3.53) e ingenierías y tecnologías (RM=1.83, IC 95\% 1.01-3.30). No se encontraron diferencias significativas en los momios de los estudiantes de nuevo ingreso a la UAZ en el grupo de 18 años, de las áreas de ciencias socio-políticas económicas y administrativas, ciencias básicas de Zacatecas-Guadalupe y otros estados, que no realizaban actividad física y sí consumían alcohol y tabaco (Tabla 4).

Tabla 4. Razones de momios ajustadas de sobrepeso por características generales de los alumnos de nuevo ingreso a las licenciaturas de la Universidad Autónoma de Zacatecas, 2015 ( $n=3972$ )

\begin{tabular}{|c|c|c|c|c|}
\hline & $\mathbf{F}$ & $\begin{array}{l}\text { Razón de } \\
\text { Momios" }\end{array}$ & $95 \%$ IC & $\mathbf{p}$ \\
\hline \multicolumn{5}{|l|}{ Género } \\
\hline Hombre & 1,740 & 1.22 & $1.02-1.45$ & $0.025^{*}$ \\
\hline Mujer & 2,232 & & & \\
\hline \multicolumn{5}{|l|}{ Edad } \\
\hline$\geq 19$ años & 1700 & 1.36 & $1.02-1.82$ & $0.033^{*}$ \\
\hline 18 años & 1899 & 0.93 & $0.70-1.24$ & 0.647 \\
\hline$\leq 17$ años & 373 & & & \\
\hline \multicolumn{5}{|l|}{ Áreas académicas } \\
\hline Ciencias de la salud & 1,385 & 1.88 & $1.05-3.35$ & $0.032 *$ \\
\hline Ciencias sociales & 629 & 1.93 & $1.06-3.48$ & $0.030 *$ \\
\hline Cs Sociopol, económ.y admvas. & 570 & 1.80 & $0.99-3.26$ & 0.054 \\
\hline Humanidades y educativas & 311 & 1.90 & $1.01-3.53$ & $0.044^{*}$ \\
\hline Ingenierías y tecnologías & 809 & 1.83 & $1.01-3.30$ & $0.045^{*}$ \\
\hline Ciencias básicas & 149 & 1.95 & $0.98-3.84$ & 0.054 \\
\hline
\end{tabular}


Tabla 4. Continuación.

\begin{tabular}{|c|c|c|c|c|}
\hline & $\mathbf{F}$ & $\begin{array}{l}\text { Razón de } \\
\text { Momios" }\end{array}$ & $95 \%$ IC & $\mathbf{p}$ \\
\hline Arte y cultura & 119 & & & \\
\hline \multicolumn{5}{|l|}{ Procedencia } \\
\hline Zac-Guadalupe & 1,830 & 1.07 & $0.90-1.25$ & 0.428 \\
\hline Otros estados & 139 & 1.07 & $0.69-1.63$ & 0.771 \\
\hline Estado Zac & 1,979 & & & \\
\hline \multicolumn{5}{|l|}{ Actividad física } \\
\hline No & 1,551 & 1.13 & $0.96-1.32$ & 0.141 \\
\hline $\mathrm{Si}$ & 2,421 & & & \\
\hline \multicolumn{5}{|l|}{ Consumo de alcohol } \\
\hline $\mathrm{Si}$ & 1,728 & 1.08 & $0.91-1.28$ & 0.377 \\
\hline No & 2,126 & & & \\
\hline \multicolumn{5}{|l|}{ Consumo de tabaco } \\
\hline $\mathrm{Si}$ & 549 & 1.15 & $0.91-1.45$ & 0.219 \\
\hline No & 3,257 & & & \\
\hline
\end{tabular}

\#Ajustado por sexo, edad, áreas académicas, procedencia, actividad física, consumo de alcohol y tabaco usando regresión logística.

*Significancia estadística.

\section{Discusión}

En este trabajo, se analizó la información de cerca del $80 \%(n=3,972)$ de los alumnos de nuevo ingreso, a 33 de las 40 carreras que ofrece la Universidad Autónoma de Zacatecas. La mayoría de los estudiantes se encuentran entre los 17 y 24 años, una edad en la que se producen varios cambios clave en la vida, especialmente en lo que respecta a la autonomía en la elección de sus hábitos y estilos de vida ${ }^{21}$. En un estudio realizado por Muñoz-Cano et al, en estudiantes de nuevo ingreso a la Universidad Juárez Autónoma de Tabasco en 2011, los participantes fueron 3,559 alumnos, de los cuales 2,040 eran mujeres (57.1\%) y 1,519 hombres (42.9\%), en el área de ciencias de la salud tuvieron al mayor número de estudiantes $(\mathrm{n}=862)^{22}$. Como se ha mencionado anteriormente, la UAZ está constituida por siete áreas académicas en el nivel superior, en la que predominan las mujeres $(n=2,232)$ que representan el $56.1 \%$ con respecto a los hombres $(n=1,740)$ con el $43.8 \%$. El área de ciencias de la salud $(n=1,385)$ al igual que en el estudio de Muñoz-Cano et al, es el de mayor número de estudiantes.

Los cambios en el estilo de vida y la alimentación, como lo mencionan en su artículo Lorenzini et al, ha dado lugar a una creciente prevalencia de sobrepeso y obesidad ${ }^{5}$. En México, de acuerdo a los resultados de la ENSANUT 2016, en el grupo de adolescentes de 12 a 19 años de edad la prevalencia combinada de sobrepeso y obesidad fue de $36.3 \%{ }^{8}$. Por esto, llevaron a cabo un estudio en 178 estudiantes universitarios de nuevo ingreso a la Universidad Autónoma de Yucatán, ubicada en la ciudad de Mérida, en donde encontraron que el sobrepeso y obesidad clase 1 y obesidad clase 2 , representaron el $48.87 \% \%^{5}$, 12.5 puntos porcentuales por arriba de la media nacional. Otro estudio realizado por Rangel-Caballero et al, determinaron el exceso de peso por medio del IMC en $26.47 \%$ de los estudiantes de recién ingreso a una universidad privada de Bucaramanga-Colombia, durante el periodo comprendido entre agosto y diciembre de 2013, con una prevalencia de sobrepeso del $20.26 \%$ y de obesidad del $6.21 \%{ }^{7}$. En la UAZ "Francisco García Salinas", en el 2013 se realizó un estudio para determinar el IMC, y se encontró que el $21.5 \%$ de los estudiantes universitarios cursaba con sobrepeso y más del $10 \%$ presentaba algún grado de obesidad ${ }^{23}$. En nuestro estudio, esta prevalencia de sobrepeso fue de $22.2 \%$ y obesidad de $8.44 \%$, la prevalencia combinada en la UAZ es del 30.7\%, 5.6\% menor que la prevalencia reportada a nivel nacional por parte de la ENSANUT 2016, para este grupo de edad. 
Con respecto a la práctica regular de actividad física, Mantilla-Toloza et al, en su estudio realizado a 548 estudiantes universitarios de nuevo ingreso a las titulaciones de Fisioterapia, Enfermería, Medicina, Psicología y Magisterio, de la Universidad de Murcia, España, señalan que los hombres realizan actividad física en $87 \%$ y las mujeres en $78 \%{ }^{24}$. Con respecto a lo encontrado en nuestro estudio, se puede ver que la población de nuevo ingreso a la UAZ, se ubica por debajo, en cuanto a la realización de actividad física regular (hombres 73.5\%; mujeres 51.1\%). Por otro lado, es importante mencionar que el $37 \%$ de los estudiantes son sedentarios de acuerdo al estudio realizado por González-Sandoval et al, en un grupo de 620 alumnos del programa educativo de Farmacobiología del Centro Universitario de Ciencias Exactas e Ingenierías (CUCEI) de la Universidad de Guadalajara, México ${ }^{25}$. Estos resultados son consistentes con los hallados en nuestro estudio que indican que el 39.0\% de los estudiantes de recién ingreso de la UAZ son sedentarios. Esto representa un área de oportunidad para la promoción de actividad física, por parte del Departamento de Actividad Física (DAF) de la universidad.

En otro estudio de Martínez-Pastor et al, seleccionaron a 1,179 estudiantes de la Universidad Católica San Antonio de Murcia, de primero y último grado, donde observaron que los estudiantes fumadores de primer grado representaron el $36.4 \%{ }^{26}$ significativamente mayor que el $14.4 \%$ encontrado en los alumnos de nuevo ingreso a la UAZ. De igual manera, en el mismo estudio de Mantilla-Toloza et al ${ }^{24}$, se encontró que los hombres resultan ser más fumadores (46\%) que las mujeres (41\%), además de que los hombres (62\%) consumen más alcohol que las mujeres (57\%). Al comparar estos resultados con los observados en los universitarios de nuevo ingreso a la UAZ, se tiene que los hombres también son más fumadores $(20.8 \%)$ que las mujeres $(9.5 \%)$ y consumen en proporción $(58.3 \%)$, más alcohol que las mujeres (34.3\%). Una de las limitaciones del estudio en cuanto a la variable "consumo de drogas" fue que, al tratarse de una historia clínica para requisito de ingreso a la universidad, la pregunta no fue contestada o fue una respuesta subreportada por los estudiantes, por lo que fue eliminada del análisis.

Finalmente, encontramos que existe un mayor riesgo de presentar sobrepeso si se es hombre, se tiene 19 años, se forma parte de las áreas académicas de ciencias de la salud, ciencias sociales, humanidades y educativas e ingenierías y tecnologías.

\section{Conclusión}

Este estudio nos proporciona información muy valiosa de los estudiantes universitarios de nuevo ingreso a las licenciaturas e ingenierías de la UAZ, en el cual se revisa el estado nutricional, algunas de las características sociodemográficas y los estilos de vida saludables y no saludables. Para atacar este problema, dentro de la universidad se puede contribuir a reducir las prevalencias de sobrepeso y obesidad de los estudiantes a través de intervenciones dirigidas, específicamente, a modificar las conductas de riesgo, mejorar la alimentación y promover estilos de vida que logren disminuir los problemas de salud a corto y mediano plazo. Además, los estudiantes se encuentran en un entorno de aprendizaje y aún están en una edad en la que se pueden mejorar los comportamientos de salud, ya que estos tienen un impacto en la salud más adelante en la vida.

\section{Responsabilidades éticas}

Protección de personas y animales. Los autores declaran que para esta investigación no se han realizado experimentos en seres humanos ni en animales.

Confidencialidad de los datos. Los autores declaran que han seguido los protocolos de la Clínica Universitaria-UAZ, sobre la confidencialidad de la publicación de datos de los pacientes.

Derecho a la privacidad y consentimiento informado. Los autores declaran que en este artículo no aparecen datos de pacientes.

Financiamiento. Ninguno.

Conflicto de intereses. Los autores declaran no tener ningún conflicto de intereses. 


\section{Referencias}

1. Pérez-Ugidos G, Lanío FA, Zelarayán J, et al. Actividad física y hábitos de salud en estudiantes universitarios argentinos. Nutr. hosp. 2014; 30(4): 896-904.

http://dx.doi.org/10.3305/nh.2014.30.4.7641

2. Hernández A, Singh P, Andino C, et al. Caracterización de hábitos relacionados con enfermedades crónicas en población universitaria de Honduras. RCSP. 2015; 41 (2): 324-34.

3. Valdes-Badilla P, Godoy-Cumillaf A, Herrera-Valenzuela T, et al. Comparación en hábitos alimentarios y condición física entre estudiantes de educación física y otras carreras universitarias. Nutr. hosp. 2015; 32(2): 829-36. http://dx.doi.org/10.3305/nh.2015.32.2.9194

4. Pengpid S, Peltzer K, Kumar-Kassean H, et al. Physical inactivity and associated factors among university students in 23 low-, middle- and high-income countries. Int J Public Health. 2015; 60(5): 539-49. http://dx.doi.org/10.1007/s00038-015-0680-0

5. Lorenzini R, Betancur-Ancona DA, Chel-Guerrero LA, et al. Estado nutricional en relación con el estilo de vida de estudiantes universitarios mexicanos. Nutr. hosp. 2015; 32(1): 94-100. http://dx.doi.org/10.3305/nh.2015.32.1.8872

6. Yahia N, Achkar A, Abdallah A, et al. Eating habits and obesity among Lebanese university students. Nutr J. 2008; 7(32): 6pp. http://dx.doi.org/10.1186/1475-2891-7-32

7. Rangel-Caballero LG, Rojas-Sánchez LZ, Gamboa-Delgado EM. Sobrepeso y obesidad en estudiantes universitarios colombianos y su asociación con la actividad física. Nutr. hosp. 2015; 31 (2): 629-36. http://dx.doi.org/10.3305/nh.2015.31.2.7757

8. Ruíz-Matus C, Gaona-Pineda EB, Gómez-Humarán IM, et al. Encuesta Nacional de Salud y Nutrición de Medio Camino 2016. Cuernavaca: Instituto Nacional de Salud Pública; 2017.

9. Riba i-Sicart M. Estudio de los hábitos alimentarios en población universitaria y sus condicionantes. [Tesis de doctorado]. Barcelona: Universitat Autónoma de Barcelona; 2002.

10. Strunin L, Díaz-Martínez A, Díaz-Martínez LR, et al. Changes in Alcohol Use Among First Year University Students in Mexico. Subst Use Misuse. 2015; 50(1): 106-13. http://dx.doi.org/10.3109/10826084.2014.960591

11. Sánchez-Socarrás V, Aguilar-Martínez A. Hábitos alimentarios y conductas relacionadas con la salud en una población universitaria. Nutr. hosp. 2015; 31(1): 449-57. https://doi.org/10.3305/nh.2015.31.1.7412

12. Deforche B, Van Dyck D, Deliens T, et al. Changes in weight, physical activity, sedentary behaviour and dietary intake during the transition to higher education: a prospective study. Int J Behav Nutr Phys Act. 2015; 12(16): 10pp. http://dx.doi.org/10.1186/s12966-015-0173-9

13. Papadaki A, Hondros G, Scott-J A, et al. Eating habits of university students living at, or away from home in Greece. Appetite. 2007; 49(1): 169-76. https://doi.org/10.1016/j.appet.2007.01.008

14. Bagordo F, Grassi T, Serio F, et al. Dietary habits and health among university students living at or away from home in Southern Italy. J food nutr res. 2013; 52(3): 164-71.

15. Musaiger AO, Awadhalla MS, Al-Mannai M, et al. Dietary habits and sedentary behaviors among health science university students in Bahrain. Int J Adolesc Med Health. 2017; 29(2). http://dx.doi.org/10.1515/ijamh-2015-0038

16. Lema-Soto LF, Salazar-Torres IC, Varela-Arévalo MT, et al. Comportamiento y salud de los jóvenes universitarios: satisfacción con el estilo de vida. Pensam. psicol. 2009; 5(12): 71-87.

17. Sánchez-Ojeda MA, De Luna-Bertos E. Hábitos de vida saludable en la población universitaria. Nutr. hosp. 2015; 31(5): 1910-9. http://dx.doi.org/10.3305/nh.2015.31.5.8608

18. Argimon-Pallás JM, Jiménez-Villa J. Métodos de investigación clínica y epidemiológica. 3a ed. Barcelona: Elsevier; 2012. 
19. González-Deschamps E, Palmeros-Exsome C, Villanueva-Sánchez J, et al. Prevalencia de síndrome metabólico y su asociación con el índice de masa corporal en universitarios. Med. clin. 2007; 129(20): 766-9. https://doi.org/10.1157/13113766

20. StataCorp. 2015. Stata Statistical Software: Release 14. College Station, TX: StataCorp LP. [consultado 5 septiembre 2015]. Disponible en: https://www.stata.com/stata14/

21. Tam R, Yassa B, Parker H, et al. University students' on-campus food purchasing behaviors, preferences, and opinions on food availability. Nutrition. 2017; 37: 7-13.

http://dx.doi.org/10.1016/j.nut.2016.07.007

22. Muñoz-Cano JM, Córdova-Hernández JA, del Valle-Leveaga D. El índice de alimentación saludable de estudiantes de nuevo ingreso a una universidad de México. Nutr. hosp. 2015; 31(4): 1582-8. http: / /dx.doi.org/10.3305/nh.2015.31.4.8401

23. Mollinedo-Montaño FE, Trejo-Ortiz PM, Araujo-Espino R, et al. Índice de masa corporal, frecuencia y motivos de estudiantes universitarios para realizar actividad física. Educación Médica Superior. 2013; $27(3)$ : 189-99.

24. Mantilla-Toloza SC, Gómez-Conesa A, Hidalgo-Montesinos MD. Actividad física, tabaquismo y consumo de alcohol, en un grupo de estudiantes universitarios. Rev. Salud Pública. 2011; 13(5): 748-58. http://dx.doi.org/10.1590/S0124-00642011000500003

25. González-Sandoval CE, Díaz-BurkeY, Mendizabal-Ruiz AP, et al. Prevalencia de obesidad y perfil lipídico alterado en jóvenes universitarios. Nutr. hosp. 2014; 29(2): 315-21. http://dx.doi.org/10.3305/nh.2014.29.2.7054

26. Martínez-Pastor A, Balanza-Galindo S, Leal-Hernández M, et al. Relación entre el consumo de tabaco y alcohol y el ejercicio físico con el paso por la universidad. Aten. prim. 2009;41 (10):558-63. http://dx.doi.org/10.1016/j.aprim.2009.01.006 